\title{
Comunidad, modernidad y campesinado: reflexiones filosóficas a partir de un conflicto territorial
}

\section{Community, Modernity and Peasantry: Philosophical Reflections from a Territorial Conflict}

Gabriel Liceaga ${ }^{1}$

Fecha de recepción: 28 de junio de $2018 \quad$ Fecha de aceptación: 11 de diciembre de 2018

\section{Resumen}

Este artículo trata acerca del concepto de comunidad en el contexto latinoamericano contemporáneo. Su objetivo principal es comprender qué resonancias despierta este concepto y por qué su utilización se torna recurrente, en la medida en que avanzan los procesos de modernización capitalista en curso. El punto de partida de este trabajo es una investigación acerca de un conflicto territorial que tiene lugar en la provincia de Mendoza (Argentina) desde el año 2006. Teniendo como referencia esta investigación, se articula el análisis de fuentes primarias con la consideración de procesos macro-sociales, que son aprehendidos a través del uso de categorías filosóficas. Como principal resultado, se logra un análisis empíricamente fundado de las dimensiones filosóficas del concepto de comunidad, que destaca sus proyecciones utópicas.

Palabras clave: Comunidad, Modernidad, Campesinado, América Latina, Territorio.

\begin{abstract}
This article deals with the concept of community in the contemporary Latin American context. Its main purpose is to understand the reactions to this concept and why it is being used frequently as the capitalist modernity processes advance. These thoughts emerge from a research about a specific territorial conflict in Mendoza (Argentina) since 2006. Based on this research, this article analyzes primary sources to consider macro-social processes, which are apprehended through philosophical categories. The main result of this research is an empirically-based analysis of the philosophical dimensions around the community concept, which highlights its utopian projections.
\end{abstract}

KeyWords: Community, Modernity, Peasantry, Latin America, Territory.

1. Universidad de Cuyo, Facultad de Ciencias Políticas y Sociales, Argentina, gabriel.liceaga@gmail.com






\section{Introducción}

Este artículo trata acerca del concepto de comunidad en el marco de los procesos de modernización capitalista vigentes en América Latina. Puntualmente, se intenta comprender de dónde proviene la vigencia de aquel concepto, qué resonancias despierta y por qué, mientras más avanzan los cercamientos y la explotación de la naturaleza, la utilización de este concepto se torna más necesaria y recurrente.

La ocasión para estas reflexiones proviene del análisis de un conflicto territorial protagonizado por un grupo de familias campesinas, que tiene lugar en la localidad de Los Leones (provincia de Mendoza, Argentina) desde 2006 a la fecha (Liceaga 2017). Teniendo como referencia este trabajo, se articula el análisis de fuentes primarias con la consideración de procesos macro-sociales, que son aprehendidos a través del uso de categorías filosóficas. La construcción de una filosofía latinoamericana, que no renuncie a producir una explicación totalizante y crítica de la realidad (Fernández 2003); pero que apunte también a un cambio de paradigma intercultural (Fornet-Betancourt 2001) capaz de involucrar contribuciones de otros regímenes de discurso - particularmente, de las ciencias sociales- constituye, por tanto, el locus epistémico desde el cual se articula la argumentación.

En el plano metodológico, este posicionamiento implica analizar el entramado de sentidos que se anudan en la noción de comunidad. Estos presentan diferentes niveles de objetivación e informan a las prácticas, con una eficacia también diferencial. Con lo cual, más que recurrir a una usualmente más declamada que ejercida «triangulación» teórica o metodológica, se trata al concepto de comunidad como una «configuración», en términos de la Garza Toledo (2018). En otras palabras, se apunta a dar cuenta de las modulaciones que este concepto experimenta a nivel de las prácticas, para lo cual se recurre a diferentes técnicas (entrevistas, análisis documental), fuentes y registros teóricos. Al reverso, se aspira a que este análisis contribuya a reconstruir el sentido que anima a la singular recurrencia discursiva de lo comunitario en esta y otras situaciones, para lo cual se recurren a un instrumental filosófico.

El artículo se compone de dos apartados principales y las conclusiones. En la primera parte, se presentan algunas de las principales dimensiones en las que emergió el concepto de comunidad en el análisis del conflicto de Los Leones. Se distinguen así nociones económicas, políticas y jurídicas, que se sintetizan en el concepto de «territorio comunitario». En la segunda parte se analizan otras aristas de esta cuestión, retomando y nutriendo reflexiones realizadas en otras ocasiones a propósito del concepto de comunidad (Liceaga 2013). Finalmente, en las conclusiones, se explicita el ángulo interpretativo a partir del cual se utiliza y comprende el concepto en cuestión.

\section{El territorio comunitario de Los Leones}

Como se dijo, este artículo tiene su punto de partida en el análisis de un conflicto territorial que tiene lugar en la provincia de Mendoza (Argentina) desde el año 2006. Este tiene como protagonistas a una colectividad de campesinos/as dedicados mayormente a la ganadería y a un grupo de abogados que planean desarrollar también un proyecto ganadero (bovino), pero desde un enfoque empresarial. El foco del conflicto entre ambas partes está centrado en la propiedad y posesión de un sector de un campo que ambas reivindican como propias: los primeros, en virtud de su larga presencia y actividad en la zona; los segundos, en razón de la disposición de un título de propiedad que compraron oportunamente. La existencia de estos intereses encontrados llevó, en un primer momento, al cercamiento de parte del campo -lo que generó una airada respuesta de las personas campesinas- y luego, a la judicialización del conflicto.

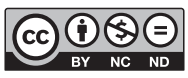


El concepto de comunidad emergió tempranamente en el análisis del conflicto de marras. En efecto, quien se acerque al campo de Los Leones verá a la orilla del camino el siguiente cartel (Figura 1):

Figura 1

Cartel en la entrada al campo de Los Leones
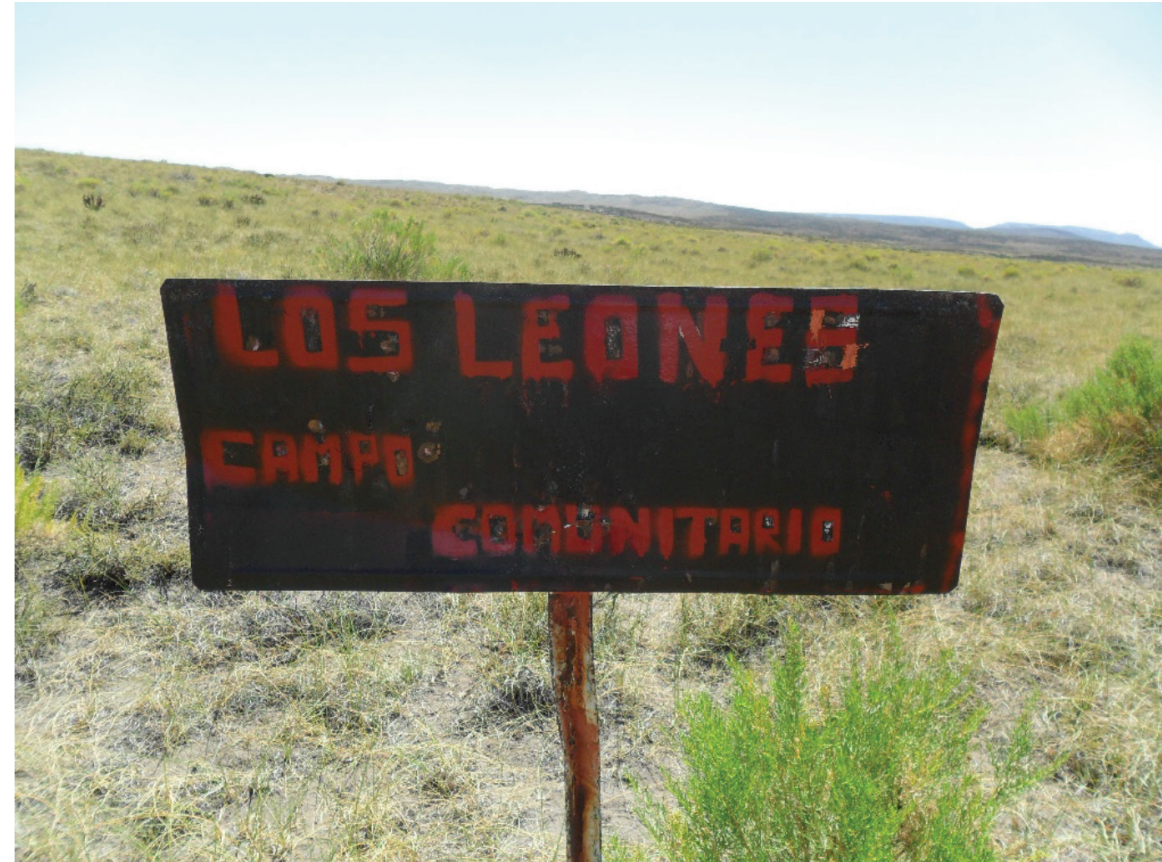

Fuente: Trabajo de Campo (Febrero de 2013)

¿Qué significa en este contexto la expresión «campo comunitario»? Podría pensarse que el cartel se limita a señalar que se está ante una forma de propiedad diferente a la propiedad privada. Sin embargo, como se verá, esta señal no se refiere únicamente a determinaciones jurídicas, sino más bien a prácticas a través de las cuales los campesinos de la zona se territorializan; es decir, se apropian simbólica, política y económicamente del espacio (Porto Gonçalves 2009; Haesbaert 2011). El territorio comunitario, de esta manera, aparece como uno de los ejes fundamentales del conflicto.

\subsection{El territorio comunitario como ámbito de la reproducción de la vida}

La principal actividad económica desarrollada por los campesinos de Los Leones, entendiendo por tal el conjunto de prácticas destinadas a la producción y reproducción de los «bienes materiales y servicios que hacen la vida posible» (Narotzky 2004), es la cría de ganado, en su mayor parte caprino; pero también ovino, bovino y equino. Esta actividad se organiza a nivel de las unidades domésticas, conformadas por familias (nucleares o ampliadas) que habitan en una misma vivienda. En el entorno rural mendocino estas unidades suelen denominarse «puestos». ${ }^{1}$ Los puestos aparecen así como unidades de producción y consumo orientados a la subsistencia, que completan en su interior la totalidad del circuito

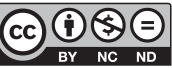


productivo, se nutren en general de mano de obra familiar y destinan la mayor parte de su producción al autoconsumo (Torres 2010). El puesto enlaza, por lo tanto, actividades ligadas a la producción y la reproducción de sus habitantes, que pueden ser individuos o familias.

Si bien cada puesto concentra y organiza gran parte de las actividades que desarrollan sus habitantes, las unidades domésticas no deben concebirse como entidades aisladas o separadas unas de otra. En efecto, los grupos de personas puesteras - puntualmente, aquel que habita en Los Leones- tienen prácticas que permiten pensar a sus unidades domésticas como englobadas dentro de lo que Raúl Prada (2014) denomina una «formación comunitaria»; o en términos más habituales, una «comunidad».

¿Qué justifica esta afirmación? Quizás el aspecto más evidente del carácter comunitario de la vida en Los Leones, tiene que ver con las formas de uso y apropiación del espacio, especialmente de las zonas de pastoreo. Al igual que ocurre en otras regiones del país, ${ }^{2}$ en Los Leones impera un régimen de acceso compartido a las pasturas. En otras palabras, los animales en Los Leones pastorean a campo abierto, sin ser delimitados por alambradas. Otros recursos además de las zonas de pastoreo (aguadas, refugios en el monte y un arroyo, que da nombre a la zona) son también compartidos por los campesinos.

De esta forma, el patrón de acceso al territorio se encuadra dentro de lo que Susana Narotzky (2004) denomina un régimen de apropiación de los recursos de carácter «comunal». Esto es, un sistema en el que los derechos se asignan de forma colectiva, y en donde el principal factor restrictivo consiste en la definición de las reglas de inclusión en la comunidad, que están sujetas a determinados criterios, entre los que sobresalen la consanguineidad, la residencia y la antigüedad en el lugar. ${ }^{3}$

La inexistencia de alambrados en las zonas de pastoreo supone, por lo tanto, no un descontrol ni una ausencia de límites en el aprovechamiento de los recursos, sino una serie de acuerdos para su acceso, e implica una comunicación constante entre los campesinos acerca de cómo organizar el cuidado de los animales y el campo. Estos acuerdos se materializan en el siguiente croquis, dibujado por uno de los campesinos, en donde se indican las zonas donde suelen ser conducidos los animales de cada puesto: 
Figura 2

Zonas de pastoreo en Los Leones

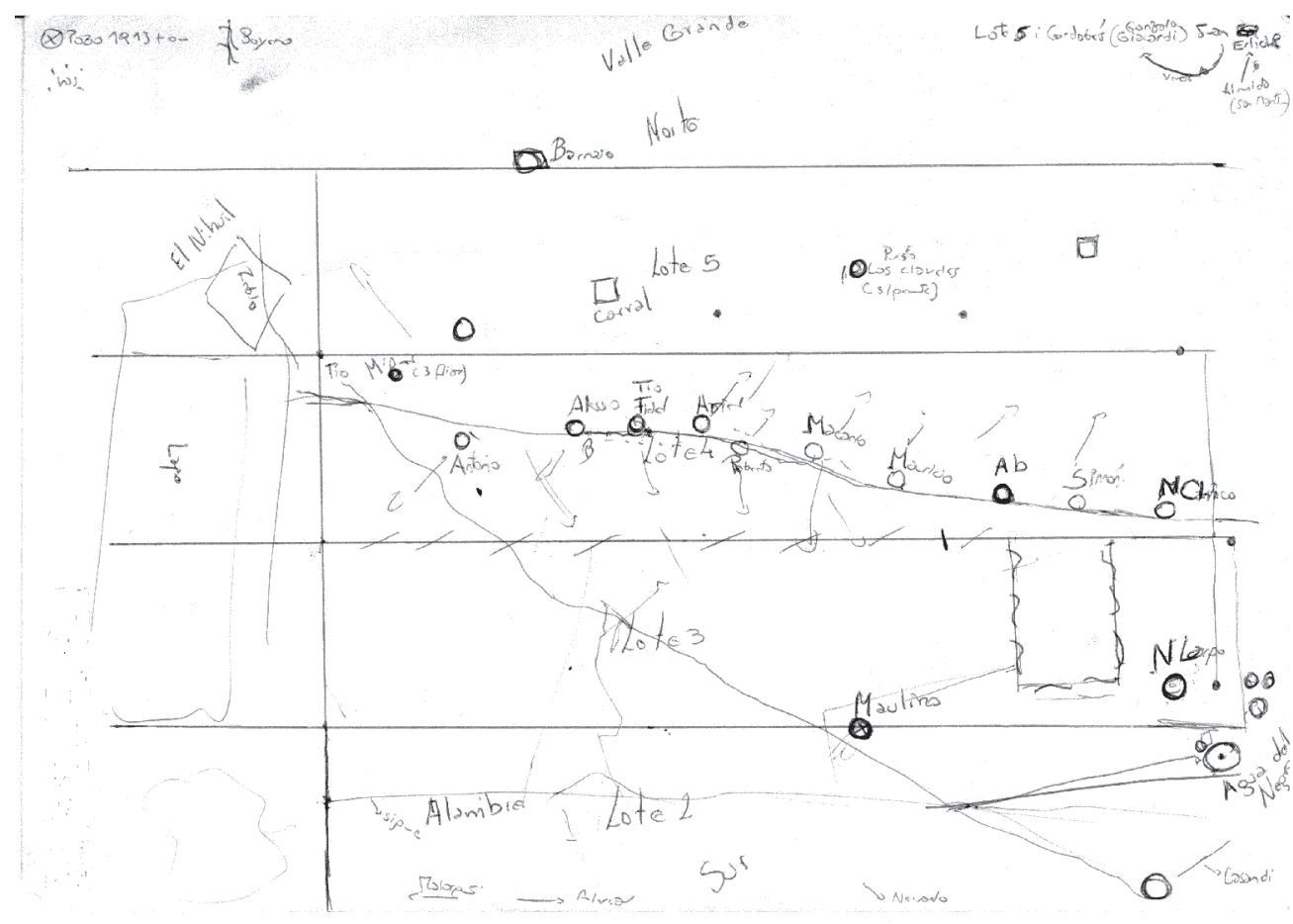

Fuente: Trabajo de Campo, (julio de 2012).

Como se ve en la Figura 2, la mayor parte de los puestos (marcados con un círculo) están alineados sobre un arroyo (línea sinuosa en el cuadrante central). Las flechas, hacia arriba y hacia abajo, indican las zonas de pastoreo habituales de los animales de cada puesto. Como se ve, existe un orden y una gestión del recurso forrajero que deriva de acuerdos tácitos, y que se expresa en el conocimiento que los puesteros tienen acerca de dónde se encuentran los animales propios y ajenos.

Otra costumbre en la que se evidencia el carácter comunitario de la territorialidad de Los Leones son las marcas del ganado bovino. Este evento se realiza una vez al año (en el mes de marzo), y consiste en agrupar a la totalidad de las vacas de los diferentes puestos en un solo corral, para que cada puestero le imprima su señal. El origen de esta costumbre reside en la necesidad de determinar la propiedad de los bovinos criados a campo abierto, en un entorno en el que no existían los alambrados. Como señala un testigo de la causa judicial en la que están involucrados los campesinos, «cualquiera de los que hemos vivido aquellas épocas de campos abiertos conocemos y hemos hecho los trabajos de esta manera, porque señalar a campo abierto animales que podían ser hijos o no hijos de la gran cantidad de puesteros que había, se consideraba una traición o un robo, por eso en los campos abiertos nadie señalaba sin la presencia de los vecinos ni marcaban tampoco» (Primer Juzgado en lo Civil, Comercial y Minas de San Rafael s.f., 415).

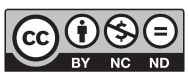


Este evento, sin embargo, no representa solo una instancia de control de la propiedad, sino que constituye también un momento de encuentro, diversión y aprendizaje ecuestre y ganadero para los niños. Durante esos días, se utilizan vestimenta e implementos tradicionales (bombachas, espuelas, botas de vino, etc.), y se comentan los chistes que se han realizado por las noches en los campamentos (Notas de campo 2016). Esta es una de las razones por las cuales, durante las marcas, no solo participan los pobladores, sino también gente de los alrededores e incluso amigos y conocidos que no tienen vacas.

Es interesante señalar que, si bien este es un encuentro abierto, existen también límites para la participación. Quienes tienen animales pero desarrollan otras formas de gestión del ganado y el territorio, más cercanas a esquemas empresariales, suelen pagar para que se marquen sus animales y no son invitados a participar. Es decir, que lo que para algunos es realizado de acuerdo con relaciones de reciprocidad (Sahlins 1983); para otros sujetos, que construyen diferentes territorialidades, es mercantilizado.

Existen otros elementos, ligados a la definición y valoración de los recursos naturales, las relaciones de reciprocidad, consanguineidad, las reglas de herencia y las migraciones, con los cuales también se pone en evidencia el carácter comunitario que revisten algunas de las prácticas económicas en Los Leones. ${ }^{4}$ No obstante, con lo dicho hasta aquí, resulta claro que algunos aspectos fundamentales del proceso de apropiación (territorialización) del espacio geográfico se dan a nivel colectivo y no familiar, mucho menos individual.

\subsection{Territorio comunitario y subjetivación política}

Es como una planta. Capaz que si hubiéramos estado de cinco años, la raíz que habríamos echado en cinco años la habrían vencido (...) Pero con cuarenta años en el lugar y toda la gente es como que pensaron voltear la planta de la raíz que habíamos echado y no fue así porque teníamos 3 veces más o cuatro que lo que ellos estaban ahí. Conocíamos el lugar, lo defendíamos porque lo queremos, porque lo necesitamos.

(Ariel, entrevista, julio de 2012)

En el marco de la disputa por sus tierras que mantienen los pobladores de Los Leones, han emprendido diversas acciones colectivas, entre las que sobresale su adscripción a una organización campesina de mayor porte (la Unión de Trabajadores Rurales sin Tierra, en adelante UST), una sostenida acción judicial y la organización de marchas y movilizaciones. Estas prácticas, sin embargo, se sostienen sobre una pertinaz resistencia en el propio territorio; es decir, en la territorialización de la acción política, que ha tenido como resultado un reforzamiento de los lazos comunitarios. De esta forma, el proceso de subjetivación política atravesado por la población de Los Leones, entendiendo por ello «la producción mediante una serie de actos de una instancia y una capacidad de enunciación que no eran identificables en un campo de experiencia dado, cuya identificación, por lo tanto, corre pareja con la nueva representación del campo de la experiencia» (Rancière 2012, 52), no puede pensarse al margen de la recreación comunitaria.

En efecto, el aspecto comunitario de las relaciones sociales en Los Leones fue tanto un presupuesto como un resultado del conflicto. Cuando este comenzó, no había acuerdo ni unidad de acción

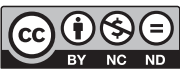


entre los campesinos, ya que mientras los pobladores de mayor edad eran más remisos y desconfiados con respecto a sus posibilidades de enfrentarse a los empresarios, los más jóvenes y las mujeres, estaban convencidos de que el campo era suyo y que debían hacer algo para defenderlo. En cualquier caso, ninguna posición o sentir era unánime: «Cuando el conflicto estábamos dispersos los puesteros. Dispersos hasta en la manera de pensar, que no todos pensábamos igual, unos queríamos defender, otros pensaban que si hacían algo iban a perder todo, que nos iban a meter en la cárcel, muchos con miedo» (Ariel, entrevista, julio de 2012).

Tras algunos intentos de organización que no dieron frutos, los/as campesinos/as convinieron en dejarse asesorar por la UST, que ya se había puesto al tanto del conflicto. A partir del involucramiento de esta organización, se comenzaron a idear vías jurídicas y políticas para enfrentar la amenaza sobre el territorio, que era cada vez mayor. Particularmente, se trabajó, junto a las abogadas de esta organización, para dar a conocer los derechos que asistían a los pobladores. Esto contribuyó a reafirmar y extender la voluntad de lucha entre todos los campesinos, que hasta ese momento era más limitada. Como señalaba una campesina en una entrevista televisiva: «Entonces nos unimos a la UST, que ellos nos dieron las riendas para empezar a luchar por lo que es nuestro» (Noticiero Popular 2008). El protagonismo de la UST en este proceso es destacado por todos los actores del conflicto (tanto sus militantes, como los/as campesinos/as y sus adversarios). Estos últimos, por ejemplo, señalaron que la UST había «concientizado con error» a los puesteros, que «les habían dado mecanismos de lucha»; aunque, desde su punto de vista, les hubieran instalado también un mensaje equivocado respecto de los derechos que realmente los asistirían (Notas de Campo 2012).

Progresivamente, y mediando el apoyo y asesoramiento de la UST, el conjunto de los/as pobladores se fue involucrando en un conjunto de prácticas que exigían la presencia en el territorio: pararse frente a las topadoras, realizar vigilias frente a las tranqueras, enfrentar verbalmente a los empleados de los empresarios que pretendían desmontar y alambrar y avisarse mediante señales de humo cuando se acercaban los alambradores (Raimundo, entrevista, junio de 2012). En esta etapa del conflicto, se destacó la participación de mujeres y jóvenes, quienes se pusieron al frente de la resistencia. Así lo destacaba, al menos, el cronista Dante Rofi en Página 12 (2008): «todos recuerdan que el día de la topadora la Chiquita, con su voz tímida, asombró a sus familiares y amigos por la verborragia y la capacidad de retruque en el momento en que la máquina y los papeles quisieron arrebatar su casa y fuente de trabajo. Pero lo que más sorprendió fue su astucia de llevar un pequeño grabador para registrar todas las amenazas y argumentaciones de los abogados».

No obstante, pese a los esfuerzos que los/as campesinos/as realizaban, las tareas de cierre seguían su curso y la Justicia no daba lugar a sus denuncias: el territorio estaba en peligro. A pesar de ello, la voluntad de resistir y, sobre todo, el reconocimiento como dueños/as y poseedores/as se fortalecían. De esta manera, cuando la Policía Rural ordenó realizar un «rodeo judicial», según el cual los campesinos debían retirar a los animales de los campesinos del lote en disputa, estos decidieron enfrentarlo.

El rodeo judicial consistía en que la Policía retiraría a los animales del campo en disputa y los entregaría del otro lado del cerco. Los campesinos no podían evitar que los policías sacaran a los animales. Más aún, ni siquiera podían entrar a esa fracción del campo sin su permiso. Sin embargo, encontraron un modo, gracias a su conocimiento del lugar, para que los animales «volvieran»: «Ellos en un principio decían que en tres días sacaban todo lo que había. Pensaban que nosotros los íbamos a ayudar. Hasta no podíamos entrar sin permiso de ellos al campo. De los milicos. En tres días, estuvieron 10 días y no sacaron nada. Al último, los milicos todos quemados, paspados, y sacaron pocazo. No fue como ellos pensaron que era. La hacían muy fácil» (Ariel, entrevista, julio de 2012).

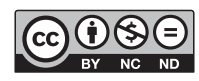

Rev. Reflexiones 98 (2) Julio-Diciembre, 2019: 113-128, E-ISSN: 1659-2859 
Tras el fracaso del rodeo judicial, los abogados contrataron a algunos guardias que comenzaron a vigilar el alambre. Pasados algunos días, estos hombres se retiraron. Una noche, hubo una fuerte tormenta, tras la cual el alambrado apareció roto; las huellas de quienes lo cortaron, borradas por el agua: «parece que la naturaleza los castigó, les llevó alambre, les llevó tranquera. Algo se lo llevó Dios y algo lo cortaron» (Notas de campo 2016). Tras ello, los animales pudieron atravesar nuevamente la frontera entre los lotes.

La rotura del alambre marcó un antes y un después en el conflicto. Su valor material y simbólico fue muy importante, constituyendo, en términos de Raúl Prada (conversación personal, 2013), una «subversión y revelación de la praxis», que reforzó el control territorial campesino y marcó un clímax en la conflictividad, quebrando, de un modo no verbal, «...el cordón sanitaire entre el discurso oculto y el público» (Scott 1990). Esta rotura puede interpretarse también como la manifestación material de la voluntad de un sujeto, que desafió, desde el territorio y colectivamente, otros poderes ejercidos en la esfera judicial. La disyuntiva entre una historia de «...envolvimiento y cierre, de "ya no es posible hacer nada", de epitafio, o bien, de apertura, de riesgo, de vida, de "es posible", opción que opera en la construcción del sí mismo y, por ende, en la construcción con el otro; o bien contra el otro» (Zemelman 2002, 32) se inclinó definitivamente hacia el segundo polo.

\subsection{El territorio comunitario como argumento jurídico-político}

En Argentina no existen, ni en la Constitución Nacional ni en el Código Civil, instrumentos jurídicos que consideren específicamente las formas campesinas de construcción del territorio (uso común de las pasturas, trashumancia, reglas de herencia, patrones de residencia patrilocales, entre otras). Como consecuencia de ello, las organizaciones campesinas se ven limitadas en sus reclamos o accionar a las figuras jurídicas consagradas por el Código Civil, pensadas dentro desde una cosmovisión liberal y poco sensible a las particularidades históricas y regionales. Otros plexos normativos, como los provenientes de la incorporación a la Constitución Nacional de tratados internacionales (por ejemplo, el convenio 169 de la Organización Internacional del Trabajo), si bien, potencialmente tienen un mayor grado de apertura hacia otras formas de propiedad y de aprovechamiento del territorio, todavía no gozan del debido reconocimiento en el sistema judicial (Romano 2011).

Pese a ello, en el marco del conflicto de Los Leones, los/as campesinos/as -en rigor, sus representantes legales- intentaron hacer uso del concepto de «posesión comunitaria», traduciendo jurídicamente las prácticas territoriales que fueron descriptas antes.

En efecto, en el marco de un recurso denominado «acción policial de posesión», interpuesto por los letrados defensores de los campesinos y destinado a resguardar su posesión del campo, estos señalaban que «los comparecientes (...) han crecido en el inmueble que poseen desde hace generaciones (...) teniendo cada uno su puesto, es decir, casas y corrales, y utilizando fuera de estas zonas individuales, una amplia superficie de los campos denominados AISOL o LOS LEONES, en forma conjunta, zona en la se encuentran las aguadas, rieles y represas, yendo el ganado en forma continua a abrevar a esas fracciones de campo poseídas en forma común por todos los accionantes desde hace décadas» (Primer Juzgado en lo Civil, Comercial y Minas de San Rafael s.f., 64).

Tras detallar algunos de los actos posesorios realizados por los campesinos en toda la extensión del territorio, se insistía en que «...las mejoras en el campo han sido realizadas en reiteradas oportunidades en forma común, ya que los lazos de vecindad y solidaridad priman en el lugar, al mismo tiempo de que son imprescindibles para la explotación de animales» (Primer Juzgado en lo Civil, Comercial y Minas de San Rafael s.f., 64). Asimismo, se enfatizaba la buena relación entre vecinos y su necesidad:

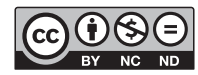

Rev. Reflexiones 98 (2) Julio-Diciembre, 2019: 113-128, E-ISSN: 1659-2859 
de que «...cada uno tiene su puesto y el campo abierto es usado por todos, de lo contrario los animales no podrían sobrevivir, es decir, es poseído por todos los actores en forma anual, pública, pacífica y continuada» (Primer Juzgado en lo Civil, Comercial y Minas de San Rafael s.f., 65).

De esta forma, el concepto de «posesión comunitaria», inexistente en el Código Civil, tomó una existencia fáctica en el cuerpo del expediente en el que se tramitó el conflicto de tierras.

Es interesante señalar que, a pesar de la debilidad jurídica de este concepto, también los abogados que se les enfrentaban se pronunciaron al respecto. Estos últimos, rechazando esta figura que los actores intentaban poner en consideración señalaban que «la propiedad comunitaria de las tierras responde a otro contexto social, antropológico, histórico, geográfico y jurídico. La propiedad comunitaria es la de las comunidades aborígenes que viven en zonas inhóspitas en condiciones de marginación social y a las que el Estado les interesa arraigar creándoles mejores condiciones de vida...» (Primer Juzgado en lo Civil, Comercial y Minas de San Rafael s.f., 237).

Tras negar la pertenencia de los campesinos a una comunidad indígena, estos cuestionaban la autenticidad de sus «sentimientos comunitarios» e incluso su adscripción a la UST aduciendo que «al menos ellos no pueden pertenecer al movimiento de los Sin Tierra, porque es un hecho incuestionable que su intención (no manifestada aún con actos posesorios) es excluyente y exclusiva, no solo para los verdaderos propietarios sino también para los co-actores (...) En realidad en ninguno de ellos anida el sentimiento comunitario» (Primer Juzgado en lo Civil, Comercial y Minas de San Rafael s.f., 236).

Resulta interesante que, en este pasaje, los demandados prescinden de un marco de referencia jurídico, dialogando directamente con la argumentación de los actores, que tiene un tinte más político. La politicidad intrínseca de la cuestión los arrastra y cuestionan, no solo su legitimidad jurídica para reivindicarse como parte de una comunidad, sino inclusive sus sentimientos.

Para finalizar, cabe mencionar que en octubre de 2013, el juez que entendía en la causa se pronunció acerca de diversos aspectos, entre ellos el de la posesión comunitaria, que desestimó en razón de la inexistencia de esta figura jurídica en el Código Civil. A pesar de ello, el fantasma de lo comunitario ya había aparecido, también en el plano jurídico, merced a la argumentación de los actores.

\section{Resonancias utópicas}

Al margen del interés que, por sí mismos, pudieran presentar los acontecimientos referentes al conflicto de Los Leones; en este artículo, estos funcionan como señal o indicio de una tendencia más general, relacionada con los procesos de modernización capitalista y el uso del concepto de comunidad. En este apartado, se trata así de pensar aquellos emergentes empíricos a la luz de una reflexión filosófica sobre las causas del resurgir del concepto de comunidad, en un contexto de profundización del capitalismo.

En efecto, como se propuso en otra ocasión (Liceaga 2013), en las luchas territoriales y ambientales que involucran particularmente a campesinos e indígenas del continente americano, continuamente aparecen referencias a temas comunitarios o a nociones cercanas como la de «bienes comunes». Los procesos de mercantilización de la tierra, el agua y el material genético, la conversión de varios tipos de derechos de propiedad (comunal, colectiva, estatal, entre otros) en propiedad privada, la supresión de formas alternativas de producción y consumo, la realización de mega emprendimientos energéticos, forestales, mineros y de infraestructura, entre otras formas de valorización capitalista en las que se verifica alguna forma de privatización de bienes sociales, culturales y naturales suele verse críticamente atravesada por la noción de comunidad. Llamativamente, no solo las resistencias a aquellos procesos

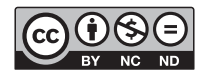

Rev. Reflexiones 98 (2) Julio-Diciembre, 2019: 113-128, E-ISSN: 1659-2859 
conceptualizados como propios de la «acumulación por desposesión» (Harvey 2004) recurren a la idea de «comunidad»; sino que, incluso, las iniciativas privadas de intervención en el marco de la responsabilidad social empresaria construyen discursiva y técnicamente a sus propias comunidades-objeto (Corporación Financiera Internacional 2007). En síntesis, la noción de comunidad, aunque parezca paradójico, resurge en una etapa histórica en la que impera y se extiende la privatización y mercantilización de los más diversos bienes públicos, sociales y naturales.

Esta aparente paradoja, encuentra un comienzo de solución cuando se observa que «comunidad» es un vocablo típico de la Modernidad, que surge, diferenciado de la idea de sociedad, en el siglo XIX europeo; y que se revitaliza actualmente ante la expansión de ciertas dinámicas «desposesorias» de la acumulación capitalista en América Latina. Esto lleva a pensar que es, precisamente en los momentos de modernización y liberalización ascendentes, cuando esta categoría resurge con mayor fuerza, ya sea en el plano teórico o político. En otras palabras, frente a formas de modernización percibidas como destructivas en lo ambiental, excluyentes en lo social y homogeneizadoras en lo cultural, se recurre a la noción de comunidad para referir tanto lo que se pretende conservar como lo que podría ser, de ponerse en marcha procesos de modernización alternativos (Liceaga 2013).

El conflicto de Los Leones no resulta una excepción a este uso -solo en apariencia- paradójico del concepto de comunidad. En efecto, entre los pliegues de las dimensiones económicas, políticas y jurídicas en las que emergió la idea de comunidad dentro de este conflicto (reseñadas en el apartado anterior), aparecieron también resonancias utópicas que conviene poner de relieve.

\subsection{Cueros, cabras y buen vivir}

«Esto nos coloca ante la necesidad de concebir a la realidad, no sujeta a regularidades, sino como una construcción permanente de la utopía por los sujetos sociales, a partir de situaciones microestructurales» (Zemelman 1989, 38)

Como se mencionó en el apartado anterior, los/a campesinos/as de Los Leones forman parte de la Unión de Trabajadores Rurales sin Tierra (UST), organización que les prestó una decisiva colaboración en diferentes momentos del conflicto. Este vínculo se vio enriquecido, no solo por la activa militancia de muchos/as campesinos/as, sino también por la energía y creatividad que estos/as imprimieron a la formulación de proyectos productivos y de comercialización. «Bueno, ellos traccionaron muchas ideas. Muchas ideas que tienen que ver con lo productivo, con avances interesantes en cuestiones alternativas, parcelas, toda la cuestión de la cadena de las cabras» (Raimundo, entrevista, junio de 2012).

En efecto, desde 2011 se han llevado adelante diversos proyectos en la zona que involucran: el comercio de cueros caprinos, chivos y cabras de refugo, ${ }^{5}$ así como también, la producción de chacinados caprinos y de fabricación de alpargatas. Algunos de estos proyectos se han extendido a otras localidades y provincias, a partir de la experiencia iniciada en Los Leones.

Quizás uno de los proyectos más importantes, por sus resultados económicos y significación política, sea el de los cueros caprinos. Hasta el año 2011, estos cueros eran comercializados en el campo y sus precios eran bajos; o eran cambiados por verduras o yerba a comerciantes que se acercaban a los puestos. Aquel año se decidió realizar una venta conjunta de cueros a una fábrica recuperada, bajo control de sus trabajadores, localizada en la periferia de la ciudad de Mendoza. El precio subió

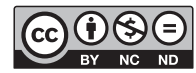


ostensiblemente, lográndose que los productores conservaran la diferencia que antes obtenían los intermediarios (Fuertes 2014).

La búsqueda de nuevos y mejores canales de comercialización también alcanzó al producto principal, los chivos o cabritos lechales. ${ }^{6}$ A partir de 2012, los campesinos comenzaron a vender parte de su producción a un frigorífico de La Pampa, mejorando los términos del intercambio en un 25\%, respecto del precio pagado en ese momento por los cabriteros que se acercaban a la zona (Fuertes 2014). Desde entonces, se han vendido también animales a través de diversas redes de comercio justo.

La sinergia lograda entre la comunidad de Los Leones, la UST y algunas instituciones públicas (particularmente, la Subsecretaría de Agricultura Familiar y el Instituto Nacional de Tecnología Industrial, INTI), permitió también la apertura de mercados para nuevos productos. Como ejemplo de ello, se puede mencionar la exportación de cabras de refugo, a través de una empresa intermediaria, a países de Medio Oriente y Centroamérica; la confección de alpargatas, a partir de cueros de cabra, y la producción de chacinados y embutidos.

En definitiva, los/as campesinos/as de Los Leones dieron el puntapié inicial a diferentes proyectos, algunos de los cuales se han extendido a otras poblaciones de fuera de la provincia. Este dinamismo se basa, no solo en su trabajo, sino también en las expectativas e ilusiones que fueron capaces de despertar en otros miembros/as de la UST. Como señala Raimundo (entrevista, junio de 2012), «después una cosa muy interesante que nos generaron a todos fue decir: bueno, existe un modelo campesino, y ¿cuál es? ¿Adónde queremos llegar? ¿Cómo queremos vivir? Y ves a los compañeros y decís: "así queremos vivir" (...) ésta es, ésta es nuestra visión, nuestra forma de vida. Con las mismas costumbres, la misma tradición, la misma forma de ser. Una expectativa con mucha lucha pero a su vez viviendo bien. Sería el "Buen Vivir", la concepción esa, en chiquitito la concepción del Buen Vivir (...) esa relación entre la tierra, la comunidad, la familia» (Raimundo, entrevista, junio de 2012).

¿Qué otros elementos aparecen en esta representación del «Buen Vivir», que, según el entrevistado, tendría lugar en Los Leones? Por un lado, la conservación de algunas tradiciones, «la vida campesina de esa zona ha quedado bastante protegida de otras cosas» (Raimundo, entrevista, junio de 2012). Pero también, como se dijo antes, su dinamismo y capacidad de innovación. Es por ello que, de acuerdo con este entrevistado, la comunidad de Los Leones «ha sido como un laboratorio (...) para desarrollar alternativas a la producción, porque es una comunidad que está organizada, porque hay juventud, hay gente con mucho ímpetu, con muchas ganas de probar cosas» (Raimundo, entrevista, junio de 2012). En esta combinación, entre la supuesta conservación de algunos rasgos culturales y el ímpetu por transformar determinadas realidades, se sustentarían la fortaleza de los/as campesinos/as.

Ahora bien, como marcaba el entrevistado en el fragmento anterior, el Buen Vivir «en chiquitito», tendría que ver con una forma de relación entre la tierra, la comunidad y la familia: «El aspecto familiar y el aspecto comunitario, como va y viene siempre en desarrollo de las actividades una cosa y la otra. No pueden defender la tierra si no es en comunidad; no pueden desarrollar algunas actividades si no es con la familia» (Raimundo, entrevista, marzo de 2016). La comunidad, de esta manera, aparece anudando una serie de significantes valiosos para el hablante, que en su conexión íntima con otros (tierra, familia) condensan una «visión», una «forma de vida».

Las resonancias tonnesianas de esta representación de lo comunitario resultan, para el lector versado en «Comunidad y Sociedad», evidentes. Solo que, esta vez, los elementos sobre los que insiste Tönnies (1947) en su célebre obra (lo «sentido», lo «antiguo», la «unidad», el «terruño») han pasado por el tamiz del Sumak Kawsay o Suma Qamaña andinos: «El Buen Vivir, el desarrollo individual, familiar y de la comunidad en consonancia con la Madre Tierra» (Raimundo, entrevista, abril de 2016). De esta

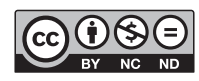

Rev. Reflexiones 98 (2) Julio-Diciembre, 2019: 113-128, E-ISSN: 1659-2859 
manera, la noción de comunidad se muestra como capaz de actualizar decimonónicas y románticas críticas del capitalismo en Europa (sintetizadas por Tönnies), con la actualización de la herencia cultural indígena, tan en boga durante las primeras décadas del siglo XXI en diversos países de América Latina. La aptitud práctica del concepto de comunidad resulta, una vez, más envidiable: la comunidad aparece como una proyección anhelada por el entrevistado (la armonía individual, familiar, social y natural), basada en prácticas efectivas (la construcción del territorio), que a su vez da lugar a acciones colectivas (proyectos productivos).

\subsection{La comunidad como ausencia presente}

La hipótesis interpretativa que guía este recorrido por los usos del concepto de comunidad es que, desde su formulación tonessiana, pasando por otros clásicos como Emile Durkheim y Max Weber, la tensión entre comunidad y sociedad constituye un intento de conceptualizar las consecuencias que los procesos de modernización capitalista tienen sobre las formas de relacionarse de las personas. De esta forma, las anheladas formas comunitarias reflejan utópicamente (aunque sobre la base de experiencias históricas concretas: la aldea campesina europea y la comunidad indígena, por ejemplo) aquello negado por la sociedad burguesa: la solidaridad, la reciprocidad, la mutua dependencia de los seres humanos en tanto seres necesitados. La comunidad puede entenderse así como una imagen utópica que refleja, como un espejo invertido, aquello que está siendo negado por la expansión de las relaciones de mercado, sobre la base de formas de sociabilidad realmente existentes en el pasado o en los márgenes de la sociedad burguesa (Liceaga 2013).

Siguiendo esta línea de razonamiento, lo que expresaría la apelación a la comunidad (ya sea a fines del siglo XIX o a principios del XXI) es una situación de crisis relacionada con la consolidación de la sociedad burguesa y las formas de racionalidad que le son características. Dicho crudamente: mientras menos comunidad hay en la realidad efectiva, o cuanto más está ella amenazada, más reaparece aquella en el plano discursivo.

Existe un filósofo contemporáneo, Franz Hinkelammert, en cuya obra pueden encontrarse contribuciones teóricas que apuntalan esta interpretación. Puntualmente, su conceptualización del sujeto como ausencia presente, es decir, como anhelo de plenitud social, tan irrealizable prácticamente como imposible de eliminar de las expectativas humanas, aparece como la clave para entender por qué se habla tanto de «comunidad», precisamente cuando esta está amenazada, pero también, en qué se apoya su particular eficacia práctica:

\footnotetext{
El llamado a ser sujeto se revela en el curso de un proceso. Por eso, el ser sujeto no es un a priori del proceso, sino resulta como su a posteriori. El ser humano como sujeto no es ninguna sustancia y tampoco un sujeto trascendental a priori (...) Se revela, entonces, que el ser sujeto es una potencialidad humana y no una presencia positiva. Se revela como una ausencia que grita y que está presente, pero lo es como ausencia. Como tal la ausencia solicita. Hacerse sujeto es responder a esta ausencia positivamente, porque esta ausencia es, a la vez, una solicitud. Se trata de una respuesta positiva a la ausencia, sin eliminarla como ausencia (Hinkelammert 2003, 496).
}

En este pasaje, Hinkelammert (2003) se refiere al sujeto en dos sentidos diferentes, aunque relacionados entre sí. El primero, como plenitud humana negada, en la cual las relaciones entre las personas serían directamente sociales y transparentes, y en la que existiría un reconocimiento completo del otro/ otra en cuanto ser necesitado y necesario. El segundo, como aquellas disposiciones activadas por el intento de responder al requerimiento utópico del sujeto.

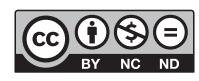

Rev. Reflexiones 98 (2) Julio-Diciembre, 2019: 113-128, E-ISSN: 1659-2859 
Ahora bien, algo similar ocurre con el concepto de comunidad: su importancia y necesidad se hace vívida y patente en la amenaza o realidad de su negación. Sin embargo al enfrentarse esa amenaza -como lo hicieron los/as campesinos/as de Los Leones, organizados en la UST- se pone en juego el segundo sentido referido; y la comunidad se vuelve un objeto deliberadamente buscado y, en alguna medida siempre imperfecta, alcanzado.

Esto explica en definitiva porqué las formas de vida comunitarias (indígenas, campesinas, ciudadanas, afrodescendientes, entre otras) suelen ser enarboladas como un vocablo de lucha que remite simultáneamente a la tradición y al futuro, a lo que fue, a lo que es y a lo que podría ser; por pueblos y organizaciones que resisten al proyecto civilizatorio de la «sociedad». La productividad política del concepto resulta también explicada: en este resurgir de lo comunitario, se ponen en juego diferentes registros culturales y políticos que se puede asociar con las más variadas tendencias ideológicas, al interior de un magma cambiante de significados, proyectos y memorias históricas.

\section{Conclusiones}

En tiempos de capitalismo global y redes sociales resuena, vaya paradoja, un término de viejo cuño, que despierta sensaciones de proximidad y calidez: «comunidad». Las fronteras políticas, académicas y disciplinares se borran a su paso, mientras que esta recurrencia semántica se ve desmentida, día a día, por la realpolitik que rige a las conciencias, las empresas y a los Estados, más pendiente de las finanzas, que de la salud del lazo social. A pesar de todo, y como lo demostró el análisis de la experiencia de Los Leones, en anhelo de comunidad resurge como resorte discursivo desde el cual desplegar subjetividades políticas y resistencias, tanto en un plano material como simbólico.

En este artículo se ha ofrecido una interpretación filosófica, fundada empíricamente, de este particular resurgir del tema comunitario. Esta interpretación no obtura otras dimensiones que pueda presentar la utilización de este concepto que, en cualquier caso, se destaca por su carácter polisémico. Sin embargo, se cree haber llamado la atención sobre un aspecto decisivo: la interpelación simbólica de lo comunitario radica no necesariamente en su fortaleza efectiva, sino en sus alcances utópicos. En un mundo en el que, como señala Prada (2012), los códigos abstractos que valorizan el significante se abren a una variabilidad asombrosa de significados, el concepto de comunidad actúa condensando anhelos y necesidades que ligan al hablante a su tierra y a sus semejantes. Captar esta pluralidad de dimensiones en su movimiento y conjunto requiere, como se ha intentado, desdibujar fronteras disciplinares y evitar toda simplificación que cristalice solo un aspecto de la cuestión.

\section{Notas}

1. Se denomina «puestos» a las viviendas típicas de los entornos áridos y semiáridos no irrigados de la República Argentina. Un puesto, en sentido general, es una casa, típicamente de adobe, champa o de ladrillos, en cuyas inmediaciones hay corrales y otras instalaciones ligadas a las prácticas pecuarias. Estos se hallan constituidos por un conjunto de construcciones y espacios con funciones diversas, edificaciones de usos complementarios y corrales ubicados en el mismo conjunto o en las cercanías. Por lo general, la accesibilidad al agua orienta y estructura el asentamiento del puesto, los pozos, tanques, cisternas, acequias o aguadas (Pastor 2004).

2. Por ejemplo, en Santiago del Estero (Paz et al. 2000; Barbetta 2009); el norte de Córdoba (Cáceres et al. 2009, Romano 2011); el noroeste de Salta (Van Dam 2008; Hocsman 2012) y el norte de

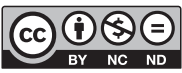


Neuquén (Bendini y Steimbreger 2010), así como también, en diversas zonas precordilleranas y cordilleranas. En Mendoza, este patrón territorial puede encontrarse a lo largo de toda la geografía provincial, fuera de los oasis de riego.

3. Desde otra perspectiva teórica, se evidencia que se cumplen en Los Leones algunas de las condiciones que Elinor Ostrom (2011) señala como propias de las gobernanzas exitosas de los bienes comunes o recursos de uso común (RUC): una comunicación fluida entre los participantes, un grado relativamente bajo de inversiones, la ausencia de reglas externas, coerciones o multas y la existencia de acuerdos de gestión que tiene a los apropiadores como protagonistas.

4. Puede trazarse, en efecto, un hilo conductor entre todas estas dimensiones. La dinámica de formación de puestos en Los Leones (y en zonas aledañas) es patrilocal, lo que significa que comúnmente es uno de los hijos varones quien hereda el puesto, mientras que el resto de los hermanos se ven obligadas a migrar. Estos, sin embargo, pueden volver a este en situaciones de dificultades económicas, y conservan también animales que se agrupan en rodeos comunes, pero cuya propiedad es diferenciada. Las relaciones de reciprocidad por lo tanto, no solo se expresan de manera generalizada (Sahlins, 1983) entre diferentes unidades domésticas, sino también al interior de cada una de ellas, en sentido intergeneracional.

5. En Argentina, se denomina «cabras de refugo» a aquellos animales de alrededor de 6 o 7 años, que han llegado al final de su ciclo productivo. Esto se evidencia principalmente en las piezas dentarias, desgastadas por la masticación, con lo cual no sólo la vejez del animal no solo está dada por su edad sino también por el tipo de forraje que consume.

6. Animales de alrededor de 2 o 3 meses de edad.

\section{Referencias}

Barbetta, Pablo. 2009. «En los bordes de lo jurídico. Conflictos por la tenencia legal de la tierra en Santiago del Estero». Tesis de doctorado, Universidad de Buenos Aires.

Bendini, Mónica, y Norma Steimbreger. 2010. «Dinámicas territoriales y persistencia campesina: redefinición de unidades y espacios de trabajo de los crianceros en el norte de la Patagonia». Revista Transporte y Territorio, 3 (julio-diciembre): 59-76.

Cáceres, Daniel, Felicitas Silvetti, Guillermo Ferrer, Gustavo Soto y Catalina Bisio. 2009. «Agriculturización y estrategias campesinas en el Norte de la provincia de Córdoba», acceso el 10 de noviembre de 2018. http://agro.unc.edu.ar/ extrural/Caceres.pdf

CFI (Corporación Financiera Internacional). 2007. Relaciones con la comunidad y otros actores sociales: manual de prácticas recomendadas para las empresas que hacen negocios en mercados emergentes. Washington DC: IFC - Grupo del Banco Mundial.

Primer Juzgado en lo Civil, Comercial y Minas de San Rafael (s.f.). Expediente 117523. Tribunales de San Rafael, San Rafael, Mendoza.

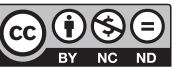


Fernández, Estela. 2003. «Los estudios poscoloniales y la agenda de la filosofía latinoamericana actual». Revista Herramienta, 8 (primavera-verano): 93-113. https://www.herramienta.com.ar/ articulo.php?id=217

Fornet-Betancourt, Raúl. 2001. Transformación intercultural de la filosofía. Bilbao, España: Desclée de Brouwer.

Fuertes, Gimena. 2008. «Los primeros pasos». Página 12, 8 de febrero. https://www.pagina12.com.ar/ diario/suplementos/las12/13-3897-2008-02-08.html

Haesbert, Rogerio. 2012. O mito da desterritorializaçao. Do "Fim dos Territórios" à Multiterritorialidade. Rio de Janeiro, Brasil: Bertrand Brasil.

Harvey, David. 2004. El Nuevo imperialismo. Madrid, España: Akal.

Hinkelammert, Franz. 2003. El sujeto y la ley. Heredia, Costa Rica: EUNA.

Hocsman, Luis Daniel. 2012. Estrategias territoriales, recampesinización y etnicidad en los andes de argentina. Ciudad de México, México: UAM Xochimilco.

Liceaga, Gabriel. 2013. «El concepto de comunidad en las ciencias sociales latinoamericanas. Apuntes para su comprensión». Cuadernos Americanos, 145: 57-85. México: Centro de Investigaciones sobre América Latina y el Caribe.

Liceaga, Gabriel. 2017. «Territorialidad y resistencias campesinas. El conflicto de "Los Leones" (Mendoza, Argentina)». Tesis de doctorado, Universidad Nacional de Córdoba.

Narotzky, Susana. 2004. Antropología económica. Nuevas tendencias. Barcelona, España: Editorial Melusina.

Noticiero Popular. 2008. El Campo Profundo. Mendoza: Noticiero Popular N², abril. Formato DVD.

Ostrom, Elinor. 2011. El gobierno de los bienes comunes. La evolución de las instituciones de acción colectiva. Ciudad de México, México: FCE, UNAM.

Pastor, Gabriela. 2004. «Patrimonio, vivienda y agua en el paisaje del noreste mendocino». Ponencia presentada en El agua en Iberoamérica. Uso y gestión del agua en tierras secas, Vol 11, El agua en Iberoamérica. Programa Iberoamericano de Ciencia y Tecnología para el Desarrollo (CYTED), Mendoza. 9-11 junio.

Paz, Raúl, Ramón Álvarez y Lastenio Castaño. 2000. «Parámetros técnico-productivos y tipologías en los sistemas caprinos tradicionales en áreas de secano». Archivo Latinoamericano de Producción Animal, 8 (2): 59-68.

Porto Gonçalves, Carlos Walter. 2009. Territorialidades y lucha por el territorio en América Latina. Geografía de los movimientos sociales en América Latina. Caracas, Venezuela: Instituto Venezolano de Ciencia y Tecnología (IVIC).

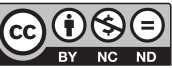


Prada, Raúl. 2012. «La colonialidad como malla del sistema-mundo capitalista Apuntes para una crítica de la economía política generalizada», acceso el 10 de noviembre de 2018, http://www.rebelion. org/noticia.php?id=159062

Prada, Raúl. 2014. «Horizontes de los mundos posibles. ¿De qué se trata?». Revista Alter-nativa, 1: 108127. http://ppct.caicyt.gov.ar/index.php/alter-nativa/article/view/3864/pdf

Rancière, Jacques. 2012. El desacuerdo. Política y filosofía. Buenos Aires, Argentina: Nueva Visión.

Rofi, Dante. 2014. «Campesinos de Mendoza agregan valor a la producción caprina». La Nación, 1 de febrero. http://www.lanacion.com.ar/1660070-campesinos-de-mendoza-agregan valor-a-la-produccion-caprina

Romano, Mariana. 2011. «Nosotros siempre fuimos campo abierto, conflictos territoriales, derechos a la tierra y poder judicial en el norte de Córdoba». Tesis de doctorado, Universidad Nacional de Córdoba, Argentina.

Sahlins Marshall. 1983. Economía de la edad de piedra. Madrid, España: Akal.

Scott, James. 1990. Los dominados y el arte de la resistencia. Ciudad de México, México: Ediciones Era.

Tönnies, Ferdinand. 1947. Comunidad y sociedad. Buenos Aires, Argentina: Losada.

Torres, Laura. 2010. «Claroscuros del desarrollo sustentable y la lucha contra la desertificación: las racionalidades económicas en el ojo de la tormenta. Estudio de caso con productores caprinos de tierras secas (Mendoza, Argentina)». Mundo Agrario, 11 (segundo semestre). Universidad Nacional de La Plata. https://www.mundoagrario.unlp.edu.ar/article/view/v11n21a11/374

Van Dam, Chris. 2008. Tierra, territorio y derechos de los pueblos indígenas, campesinos y pequeños productores de Salta. Buenos Aires, Argentina: Secretaría Agricultura, Ganadería, Pesca y Alimentos.

Zemelman, Hugo. 1989. De la historia a la política. La experiencia de América Latina. Ciudad de México, México: Siglo XXI.

Zemelman, Hugo. 2002. Necesidad de conciencia. Un modo de construir conocimiento. Ciudad de México, México: El Colegio de México. 